\title{
Degree Distribution and Hopcount in Wireless Ad-hoc Networks
}

\author{
R. Hekmat, P. Van Mieghem \\ Delft University of Technology \\ Information Technology and Systems \\ P.O. Box 5031, 2600 GA Delft, The Netherlands \\ Email: R.Hekmat@its.tudelft.nl, P.VanMieghem@its.tudelft.nl
}

\begin{abstract}
This article is a contribution to mathematical modeling and better understanding of fundamental properties of wireless ad-hoc networks. Our focus in this article is on the degree distribution and hopcount in these networks. The results presented here are useful in the study of connectivity and estimation of the capacity in ad-hoc networks. We model a wireless ad-hoc network as an undirected geometric random graph. For the calculation of the link probability between nodes we have suggested to use a realistic radio model; the socalled log-normal shadowing model. Through a combination of mathematical modeling and simulations we have shown that the degree distribution in wireless ad-hoc networks is binomial for low values of the mean degree. Further, we have investigated the hopcount and have shown that the hopcount in wireless adhoc networks can vary between the expected values for lattice networks and random graphs, depending on radio propagation conditions.
\end{abstract}

Index Terms-Graph theory, Ad-hoc networks, Radio modeling, Degree distribution, Hopcount.

\section{INTRODUCTION}

Wireless multi-hop ad-hoc networks are formed by a group of nodes that communicate with each other over wireless channels. The nodes in a wireless ad-hoc network can be mobile. Each node can, if needed, function as relay station for routing traffic to its final destination. Ad-hoc networks are decentralized, self-organizing networks and are capable of forming a communication network without relying on any fixed infrastructure.

Because of node movements and radio signal fluctuations, the topology of the network can change from time to time. However, at any instant in time, an ad-hoc network can be considered as a graph with fixed topology. In this graph, the number of links connecting any particular node to its neighbors is called the degree of that node. An ad-hoc network is said to be connected if for any source and destination node there exists a path between them. Otherwise the network is disconnected. A disconnected network may consist of several disconnected islands or subnetworks. The hopcount specifies the number of hops on the path between a source and a destination.

Many aspects of ad-hoc networks have been studied or are under investigation by the international research community. For example, extensive work has been done in the development and optimization of ad-hoc routing protocols ([1] and [2]). Study of recent literature reveals that exact mathematical modeling of ad-hoc networks is also gaining attention ([3], [4], [5]).

This research is supported by the Towards Freeband Communication Impulse of the technology programme of the Ministry of Economic Affairs in The Netherlands.
Good modeling of ad-hoc networks is essential to investigate fundamental properties of ad-hoc networks like connectivity and average path length between source and destination nodes. This article is a contribution to mathematical modeling and better understanding of fundamental characteristics in ad-hoc networks. To be specific, here we focus on two aspects: the degree distribution and the hopcount in wireless ad-hoc networks.

Knowledge of degree distribution is required to investigate connectivity in ad-hoc networks. This relation has already been indicated in e.g. [6] and [7]. However, to our knowledge, the exact distribution of the node degree in wireless ad-hoc networks has not been determined till now. In this paper we show that the degree distribution in wireless ad-hoc network is very well approximated with a binomial distribution.

The aggregate amount of relay traffic in an ad-hoc network is linked to the expected values for the hopcount. Consequently, hopcount affects directly interference levels and the capacity of ad-hoc networks ([8], [9]). In this paper we analyze the hopcount and show that the hopcount depends on radio propagation characteristics of the environment.

Our analysis of the degree distribution and the hopcount is based on a realistic radio model that allows for random variations in received signal powers. These variation are caused by obstructions and irregularities in the surroundings of the transmitting and the receiving antennas. To our knowledge this so-called log-normal shadowing radio model [10] has not been used for the modeling of wireless ad-hoc networks before.

The structure of this paper is as follows. In section II we describe our mathematical presentation of wireless ad-hoc networks which is based on a general model for geometric random graphs. In section III we introduce the log-normal shadowing radio model to complete the geometric random graph model. In section IV the degree distribution is analyzed. Here, using simulations results we compare the degree distribution in ad-hoc networks with a binomial distribution. Variations in the hopcount, depending on radio propagation conditions, are studied in section V. Our main conclusions are summarized in section VI.

\section{GEOMETRIC RANDOM GRAPH MODEL FOR AD-HOC NETWORKS}

We start this section with a summary of main characteristics of random graphs [11]. After that we discuss why a random graph is not a suitable model to describe wireless ad-hoc network topologies, and present an alternative model, the socalled geometric random graph. 
A random graph is usually denoted by $G_{p}(n)$, where $n$ is the number of nodes in the graph and $p$ is the probability of having a link between any two nodes. The fundamental assumption in random graphs is that the presence or absence of a link between two nodes is independent of the presence or absence of any other link. So, each link may be considered to be present with independent probability $p$. The degree of a node $u$, denoted as $d_{u}$, is defined as the number of nodes connected directly to node $u$. In other words, the degree of a node is the number of neighbors of that node. In a random graph, by definition, $d_{u}$ has a binomial distribution [11]:

$$
\operatorname{Pr}\left[d_{u}=k\right]=\left(\begin{array}{c}
n-1 \\
k
\end{array}\right) p^{k}(1-p)^{n-1-k} .
$$

A wireless ad-hoc network consists of a number of nodes (radio devices) spread over a certain geographic area. Every node may be connected to one or more nodes in its vicinity. We assume that connections between nodes are two-way, undirected links. When nodes move, the topology of the network may change. However, at any given point of time there is still a well defined topology available. In wireless ad-hoc network the actual set of connections, in contrast to random graphs, depends on the geometric distance between nodes. A direct consequence of the dependency of links on the distance between nodes is that in wireless ad-hoc networks there is an increased probability of two nodes to be connected with one another when they have a common neighbor. This effect is called clustering [12]. Clustering has been observed and studied extensively for other network types like social networks [13]. In the literature, graphs with distance-dependent links between nodes are sometimes referred to as geometric random graphs [4]. For reasons explained above, we will model a wireless ad-hoc network as an undirected geometric random graph. We denote an undirected geometric random graph with $n$ nodes as $G_{p\left(r_{i j}\right)}(n)$, where $p\left(r_{i j}\right)$ is the probability of having a link between two nodes $i$ and $j$ (or $j$ and $i$ ) at distance $r_{i j}$ from each other.

In $G_{p\left(r_{i j}\right)}(n)$, the total number of edges or links between nodes is by definition:

$$
L=\sum_{i=1}^{n} \sum_{j=i+1}^{n} p\left(r_{i j}\right) .
$$

Let us assume that $n$ nodes are uniformly distributed over a certain two-dimensional area with size $\Omega$. To derive the expected number of links, $E[L]$, we have used a dissection technique and assumed that area $\Omega$ is covered with $m$ small squares (or placeholders) of size $\Delta \Omega$. If each of these squares is small enough to contain at most one node, it is proved in the appendix:

$$
E[L]=\frac{n(n-1)}{m(m-1)} \sum_{i=1}^{m} \sum_{j=i+1}^{m} p\left(r_{i j}\right),
$$

where $r_{i j}$ is the distance between two placeholders $i$ and $j$. We define the link density, $\mathcal{L}$, as the ratio between $E[L]$ and $E_{\max }=n(n-1) / 2$, the maximum number of links in a fullmesh network:

$$
\mathcal{L}=\frac{E[L]}{E_{\max }}=\frac{2}{m(m-1)} \sum_{i=1}^{m} \sum_{j=i+1}^{m} p\left(r_{i j}\right) .
$$

From this formula we see that link density is independent of the number of nodes in the network. The link density depends only on the "strength of connectivity" (defined by the function $p(r)$ ) over the area of consideration. In other words, the link density is a measure that indicates how well different parts of the area can be reached from other parts.

Knowing the expected number of links in the network, the mean degree, $E[d]$, over all nodes is by definition:

$$
E[d]=\frac{2 E[L]}{n}=(n-1) \mathcal{L} .
$$

Formulas (1) and (2) are valid for any geometric random graph $G_{p(r)}(n)$. In the following section we present a realistic radio model that leads to our suggestion for the function $p(r)$ specifically for wireless ad-hoc networks.

\section{LOG-NORMAL SHADOWING RADIO MODEL}

In radio communications, the received signal levels decrease as the distance between the transmitter and the receiver increases. This phenomenon is called path-loss. Attenuation of radio signals due to the path-loss effect has been modeled by averaging the measured signal powers over long times and over many distances around the transmitter. The averaged power at any given distance to the transmitter is referred to as the area mean power $\mathbf{P}_{a}$ (in Watts or milli-Watts). The path-loss model states that $\mathbf{P}_{a}$ is a decreasing function of distance $r$ between transmitter and the receiver and can be represented by a power law [14]:

$$
\mathbf{P}_{a}(r)=c\left(\frac{r}{r_{0}}\right)^{-\eta}
$$

In this formula $r_{0}$ is a reference distance ${ }^{1}$. Parameter $\eta$ is the path-loss exponent which depends on the environment and terrain structure and can vary between 2 in free space to 6 in heavily built urban areas [10]. The constant $c$ depends on the transmitted power, the receiver and the transmitter antenna gains and the wavelength [10]. The path-loss model is often used in the study of wireless ad-hoc networks (see e.g. [7]). However, this model could be inaccurate because in reality the received power levels may show significant variations around the area mean power. These power variations are not taken into consideration in the path-loss model. In this paper we propose a more realistic radio model for the study of wireless adhoc networks. This model is the log-normal shadowing model [10], and allows for random power variations around the area mean power. Let the received power at distance $r$ from the transmitter be denoted by $\mathbf{P}(r)$. In the log-normal shadowing model the basic assumption is that the logarithm of $\mathbf{P}(r)$ is normally distributed around the logarithmic value of the area mean power:

\footnotetext{
${ }^{1}$ This distance for low-gain antennas in $1-2 \mathrm{GHz}$ region is typically chosen to be $1 \mathrm{~m}$ in indoor environments and 100 meter or $1 \mathrm{~km}$ is outdoor environments.
} 


$$
10 \log _{10}(\mathbf{P}(r))=10 \log _{10}\left(\mathbf{P}_{a}(r)\right)+x .
$$

In this expression $x$ is a zero-mean normal distributed random variable (in $\mathrm{dB}$ ) with standard deviation $\sigma$ (also in $\mathrm{dB}$ ). The standard deviation is larger than zero and, in case of severe signal fluctuations due to irregularities in the surroundings of the receiving and transmitting antennas, measurements [10] indicate $^{2}$ that it can be as high as 12 . We notice that if we assume $\sigma$ equal to zero, the log-normal model is the same as the path-loss model. So, the path-loss model can be seen as a specific case of the more general log-normal model.

Let us assume that for correct reception of radio signals it is required that $\mathbf{P}(r)>\gamma$. The "coverage area" of node $i$ in a wireless ad-hoc network is the collection of all the points $j$ in the two-dimensional space where $\mathbf{P}\left(r_{i j}\right)>\gamma$. A node can communicate directly with nodes that fall inside its coverage area but not with other nodes. It should be noticed that the area of coverage is not an area with fixed boundaries. It can change according to the probability distribution function of the log-normal shadowing model.

To eliminate parameters not relevant for our study, we normalize variables as follows. We define $R$ as the distance where the area mean power $\mathbf{P}_{a}(r)$ is equal to $\gamma$. In other words, $\gamma=c\left(R / r_{0}\right)^{-\eta}$. If we divide $\mathbf{P}(r)$ by $\gamma$ the result is:

$$
10 \log _{10}(\mathbf{P}(r) / \gamma)=10 \log _{10}\left(\widehat{r}^{-\eta}\right)+x,
$$

where we define $\widehat{r} \triangleq r / R$ as the normalized distance. From this formula we see that the logarithm of $\mathbf{P}(r) / \gamma$ has normal distribution with the mean $10 \log _{10}\left(\widehat{r}^{-\eta}\right)$ and the variance $\sigma^{2}$. The condition for correct reception of radio signals is that the $\mathbf{P}(r) / \gamma$ is more than 1 (or the logarithm of $\mathbf{P}(r) / \gamma$ is more than zero). Therefore the probability of having a link between two nodes at normalized distance $\widehat{r}$ from each other is:

$$
\begin{aligned}
p(\widehat{r}) & =\operatorname{Pr}\left[10 \log _{10}(\mathbf{P}(r) / \gamma)>0\right] \\
& =\frac{1}{\sqrt{2 \pi} \sigma} \int_{0}^{\infty} \exp \left[-\frac{\left(t-10 \log _{10}\left(\widehat{r}^{-\eta}\right)\right)^{2}}{2 \sigma^{2}}\right] d t \\
& =\frac{1}{2}\left[1-\operatorname{erf}\left(3.07 \frac{\ln (\widehat{r})}{\xi}\right)\right], \quad \xi \triangleq \sigma / \eta
\end{aligned}
$$

In this formula $\xi$ is the ratio between the standard deviation of shadowing, $\sigma$, and the path-loss exponent, $\eta$. Low values of $\xi$ correspond with small variations of signal power around the area mean power and high values of $\xi$ correspond with stronger shadowing effects. In the extreme case of $\xi=0$, there is no shadowing effect and our model is equivalent to the path-loss model. The highest values of $\xi$ correspond with severe shadowing effects in areas with low path-loss exponent. The best way to determine the most probable value range for $\xi$ is through extensive measurements. To our knowledge this type of measurements for typical wireless ad-hoc network environments are not available yet. However, based on the

\footnotetext{
${ }^{2}$ It should be noted the measurements that we refer to has been done on lower frequencies than frequencies used in WLAN networks. If a wireless adhoc network is making use of WLAN radio modules, the range of variation in $\sigma$ could be different.
}

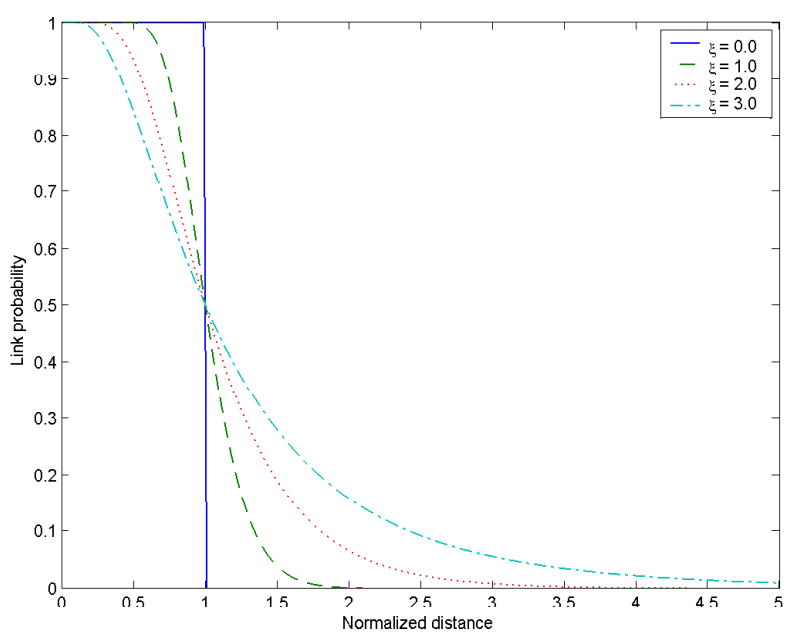

Fig. 1. Link probability with log-normal shadowing radio model as function of the normalized distance between two nodes and for different values of $\xi$.

aforementioned range of possible values for $\eta$ and $\sigma$, we note that theoretically $\xi$ may vary between 0 and 6 .

Figure 1 shows for different values of $\xi$ the link probability calculated with (3). It should be noticed that the normalized distance 1 depends directly on path-loss exponent $\eta$. So, the actual length of the normalized distance 1 for any of the lines in Figure 1 need not to be the same.

In Figure 1 we see that as shadowing becomes more severe, the link probability at short distances reduces, while at large distances the link probability increases. Physically this can be explained. With little shadowing the path-loss effect is the dominant factor, while large amount of shadowing smooths out the distance dependent path-loss effect.

Figure 2 shows results where we used (3) to located points in a squared area of size $10 \times 10$ that at a certain instant are connected to a node in the center of this area at coordinates $(0,0)$. The points shaded in this figure represent the connected points to the center node. This collection of points can be considered as the coverage area around the center node for different values of $\xi$. When $\xi=0$ (upper left subplot in Figure 2), variance of the received power around the area mean power is zero, and the coverage area is a perfect circular area with normalized radius 1 (this is just the path-loss model). As the value of $\xi$ increases, variations in the received power increase as well. Consequently we will have more nodes at normalized distances larger than 1 that may have a link with the center node. From the reduced density of shaded points at close distances to the center node, we also note that there may be nodes at distances less than the normalized distance 1 that do not have a link with the center node.

\section{A. Link density and average node degree}

Assuming normalized distances and substituting $p(r)$ in (1) with (3) provides the formula for link density with log-normal shadowing radio model, $\mathcal{L}_{\mathrm{sh}}$ : 

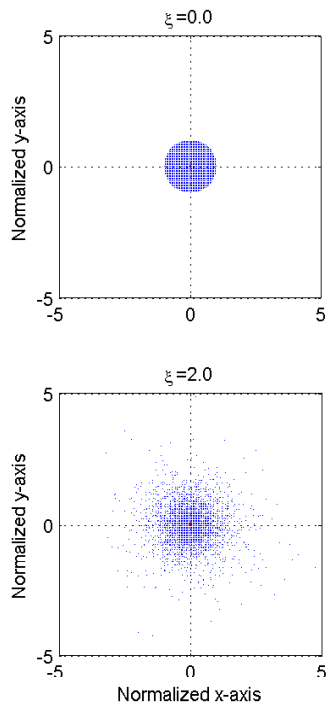
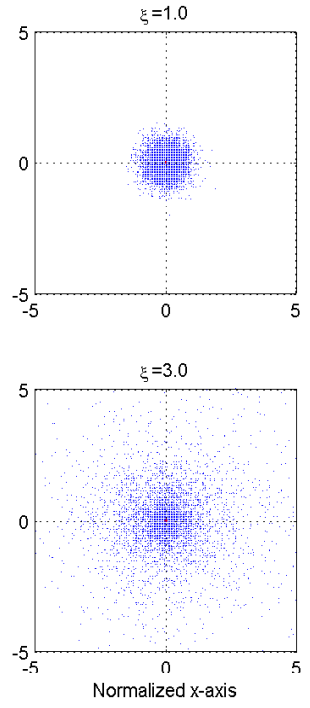

Fig. 2. Coverage of a node in the center of a service area of szie $10 \times 10$, for different values of $\xi$.

$$
\mathcal{L}_{\mathrm{sh}}=\frac{1}{m(m-1)} \sum_{i=1}^{m} \sum_{j=i+1}^{m}\left(1-\operatorname{erf}\left(3.07 \frac{\ln \left(\widehat{r}_{i j}\right)}{\xi}\right)\right)
$$

where $\widehat{r}_{i j}$ is the normalized distance between two placeholders $i$ and $j$ in the "service area" of the ad-hoc network. The service area of the ad-hoc network is the whole area where nodes are uniformly distributed. Figure 3 shows calculated values ${ }^{3}$ of the link density using (4) for different sizes of square-shaped service areas and for different values of $\xi$. Important is to notice that when the size of the service area increases, the link density tends to zero. Further, we see that the link density is higher for larger values of $\xi$. From a radio propagation point of view, a higher value of $\xi$ means a higher probability of having links with nodes at farther distances. As expected, this translates itself to a higher value of the link density over the service area.

Having the formula for the link density; the average node degree in an ad-hoc network with log-normal shadowing model follows directly from (2):

$$
E[d]_{\mathrm{sh}}=(n-1) \mathcal{L}_{\mathrm{sh}}
$$

Table 1 shows some values of the link density and the average node degree found using (4), respectively, (5); and compares them with values found through simulations. The simulation program used for verification of computed results, spreads $n$ nodes uniformly over the rectangular service area and establishes links between node pairs using the probability function (3). The simulated value of the link density in each case is the ratio of the established links to the maximum number of possible links. The simulated value of the average node degree is the mean value of the degree found for all

\footnotetext{
${ }^{3}$ In this paper wherever the link density is numerically calculated for an area of size $\Omega$, we have assumed that $\Delta \Omega=0.1 \times 0.1$. For a square-sized area of length $Z$, this means $m=10 \times Z$.
}

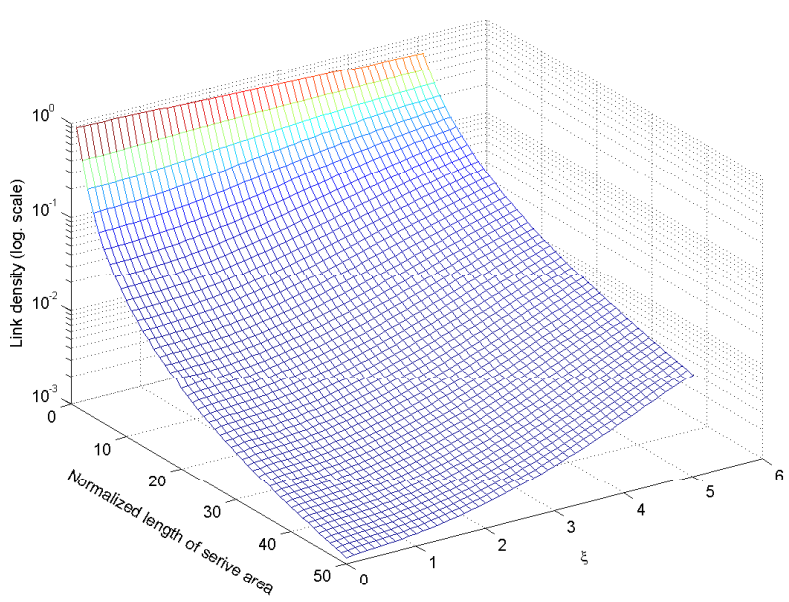

Fig. 3. Link density for square-sized areas and different values of $\xi$.

TABLE I

CALCUlATED VS SimUlated VALUES OF LINK DENSITY AND AVERAGE NODES DEGREE WITH LOG-NORMAL SHADOWING RADIO MODEL.

\begin{tabular}{|l|l|l|l|l|l|}
\hline area & $\xi$ & cal. $\mathcal{L}_{\text {sh }}$ & $\begin{array}{c}\text { cal. } E[d]_{\text {sh }} \\
n=2000\end{array}$ & $\begin{array}{c}\text { sim. } \mathcal{L}_{\text {sh }} \\
n=2000\end{array}$ & $\begin{array}{c}\text { sim. } E[d]_{\text {sh }} \\
n=2000\end{array}$ \\
\hline $5 \times 5$ & 0 & 0.1046 & 209.04 & 0.1051 & 210.02 \\
\hline $5 \times 5$ & 3 & 0.1820 & 363.73 & 0.1823 & 364.42 \\
\hline $10 \times 10$ & 0 & 0.0288 & 57.57 & 0.0287 & 57.41 \\
\hline $10 \times 10$ & 3 & 0.0606 & 121.14 & 0.0610 & 122.00 \\
\hline $20 \times 20$ & 0 & 0.0074 & 14.72 & 0.0074 & 14.85 \\
\hline $20 \times 20$ & 3 & 0.0175 & 34.98 & 0.0178 & 35.52 \\
\hline $50 \times 50$ & 0 & 0.0012 & 2.34 & 0.0012 & 2.45 \\
\hline $50 \times 50$ & 3 & 0.0030 & 6.00 & 0.0030 & 6.08 \\
\hline
\end{tabular}

nodes. It can be seen from this table that there is a good match between simulated and calculated results.

\section{Degree distribution}

In the previous section we calculated the link density and the average node degree with the log-normal shadowing radio model and verified by simulation that (4) and (5) provide accurate results, regardless of the size of the service area. In this section we concentrate on the degree distribution. For random graphs we know that by definition the degree distribution is binomial. The question considered in this section is whether the degree distribution in ad-hoc networks is binomial as well.

When nodes are uniformly distributed over the service area, for any node $i$ with an arbitrary but fixed shape of coverage area the degree distribution is binomial with a mean value that depends on the size of the coverage area of node $i$. This property follows directly from the uniform distribution and can be verified easily. However, in an ad-hoc network there are two factors that make the situation more complex. At the first place because the coverage area is determined by a probability function, the coverage area of each node does not have a fixed shape and can vary from node to node. Secondly, for nodes close to the borders of the service area, the coverage area is truncated physically by border limits of the service area. We will call the first factor "coverage probabilistic fluctuations", and the second factor "border effect". The border effect reduces the expected number of neighbors for nodes in 
the border area in comparison to nodes situated more towards the center of the service area. Taking these two aspects into account, what is distribution of the node degree in an ad-hoc network when we look at all nodes collectively?

We have investigated this question through simulations ${ }^{4}$ for different network sizes, network densities and $\xi$. Our main conclusion based on these simulations is that in wireless ad-hoc networks the node degree can be considered to be binomially distributed, with a mean value given by (5), when the "border effect" is negligible. The "coverage probabilistic fluctuations" do not seem to distort the binomial distribution of the node degree ${ }^{5}$. The border effect is negligible if: 1) the service area is much larger than coverage area of a single node and 2) the node density is low. A relatively large service area is equivalent to a low link density. Therefore, the combined effect of conditions 1 and 2 is reflected in the product of the link density and the number of nodes; in other words, in the value of average node degree (see (5)). Considering this, we can say that the border effect is negligible and the node degree is binomially distributed when the average node degree is low. In the remainder of this section we justify this statement and try to quantify the condition for its validity by simulation results.

Figure 4 shows the degree distribution found through simulations for $\xi=3$ and different number of nodes uniformly distributed over an area of $20 \times 20$. Figure 5 shows another set of simulation results found for $n=1000$ and different values of $\xi$. In both figures the solid lines represent the actual degree distribution, while in each case a dotted line shows a binomial distribution with the same mean value as the actual degree distribution. We have used the KolmogorovSmirnov test with 5\% significance level [15] to verify the hypothesis that the actual degree distribution is binomial. The Kolmogorov-Smirnov tests show that for the low values of average node degree, $E[d]$, the degree distribution is binomial with high probability. As the average node degree increases, the probability of accepting the hypothesis reduces. However, only in cases e, $\mathrm{f}, \mathrm{k}$ and $\mathrm{l}$ in Figures 4 and 5, where the average node degree is higher than 18 , the hypothesis of binomial distribution could be rejected. Other simulation results for different sizes and shapes of the service area (not presented here) are consistent with this result: in all cases where nodes are uniformly distributed over the service area, the distribution of the node degree can be considered to be binomial if the average node degree is low (lower than 18 for square-shaped areas).

In section I we mentioned that knowing the exact degree

\footnotetext{
${ }^{4}$ The simulation method is straightforward. In each simulation run we distribute $n$ nodes over an area of $x \times y$. We establish links between nodes using $p(\widehat{r})$ according to (3) and calculate the degree distribution for all nodes.

${ }^{5}$ To be able to investigate the effect of coverage probabilistic fluctuations separate from the border effect, we focused on the nodes in the inner region of the service area. The inner region of a rectangular service area of size $x \times y$ is a rectangle with size $(x-2 d) \times(y-2 d)$. The service area and the inner region are co-centered rectangles. To exclude the border effect, $d$ should be chosen in such a way that only a negligible portion of the coverage area of any node in the inner region could fall outside the service area. In our simulation for each value of $\xi$ we chose $d$ to be the distance where the link probability drops to $5 \%$ (see (3)). Our simulation results showed that regardless of the network size, the network density and the value of $\xi$, the degree distribution for inner nodes is binomial.
}
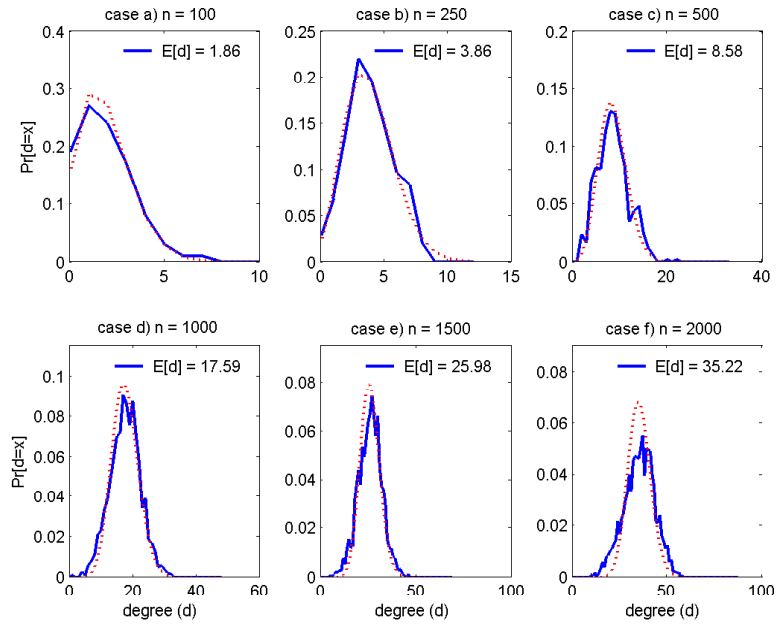

Fig. 4. Degree distribution found through simulation for different values of $n$, compared with a binomial distribution having the same mean value. Service area in all cases is $20 \times 20$ and $\xi=3$.
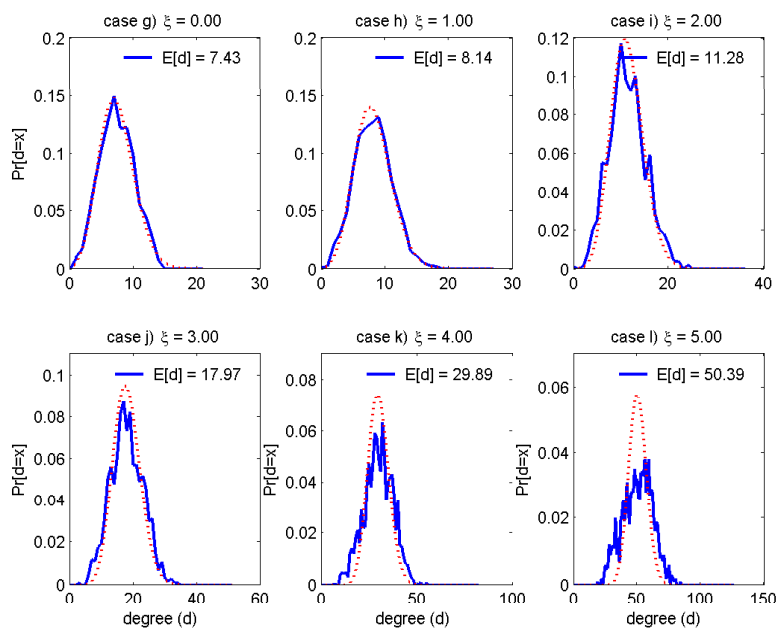

Fig. 5. Degree distribution found through simulation for different values of $\xi$, compared with a binomial distribution having the same mean value. Service area in all cases is $20 \times 20$ and $n=1000$.

distribution is relevant in the study of connectivity in adhoc networks. Without going into details we remind that the transition from disconnected to connected networks takes place at low values of mean node degree [3]. Therefore, in the regions close to the transition between connected and disconnected networks, it is save to assume that the degree distribution is binomial. In practice too, the average node degree is unlikely to be high in wireless ad-hoc networks, personal area networks or sensor networks. At the first place the transmission power of nodes forming these networks is low which limits the geographical size of the coverage area, and consequently the number of neighboring nodes. Secondly, because of medium sharing in these networks, a high node degree would result into a very low throughput per node which is an undesired situation and would be avoided.

\section{HOPCOUNT}

In this section we focus on the mean hopcount based on our geometric random graph model of ad-hoc networks. The 

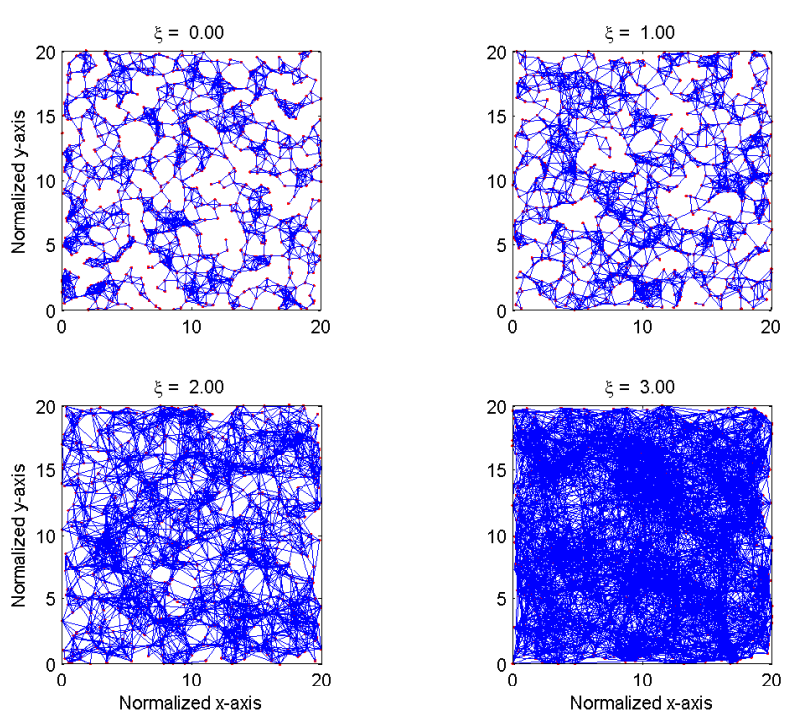

Fig. 6. Nodes and links in an ad-hoc network for different values of $\xi$. In all subplots $n=1000$, and service area is $20 \times 20$.

mean hopcount is the average distance between any pair of nodes, or the average path length. The mean hopcount in a random graph $G_{p}(n)$ with $p=E[d] / n$, is: $E[h] \sim$ $\ln (n) / \ln (E[d])$, where $E[d]$ is the average node degree in the random graph (see e.g. [12]). As opposite to random graphs where links are completely uncorrelated and relative node positions irrelevant, an extremely regulated graph in two dimensions is a rectangular lattice. Apart from border nodes, each node in a rectangular lattice has a constant degree of 4. Further, neighboring nodes are all at the same distance from each other. We call the distance between neighboring nodes the "granularity" of the lattice. The size of the lattice is the number of nodes in the lattice. The mean hopcount is of the order $O(\sqrt{n})$ in a rectangular lattice with $n$ nodes $^{6}$. For connected graphs, the mean hopcount in a lattice is higher than the mean hopcount in a random graph of the same size. In this section we show that the mean hopcount in an ad-hoc network can vary between the expected values for a lattice network and a random graph, depending on the value of $\xi$. Figure 6 visualizes the effect of the variation in $\xi$ on the topology of an ad-hoc network. When $\xi=0$, only nodes at distances less than the normalized distance 1 are connected. As $\xi$ increases, the probability of having a link between two nodes at farther distances increases as well. Consequently, the mean hopcount reduces. Figure 7 shows the hopcount distribution corresponding to each subplot in Figure 6. When the number of nodes is high enough for a giant component [3] to appear, at low values of $\xi$, the mean hopcount is close to the mean hopcount in a lattice with the same length and the same width as the service area and granularity 1 . When $\xi$ increases, the mean hopcount tends more towards the mean hopcount in a random graph with the same number of nodes and the same link probability.

\footnotetext{
${ }^{6}$ It can be proved that in a two dimensional rectangular lattice consisting of $n=k \times l$ nodes, the mean hop-count is exactly: $E[h]=\frac{k+l}{3}$.
}
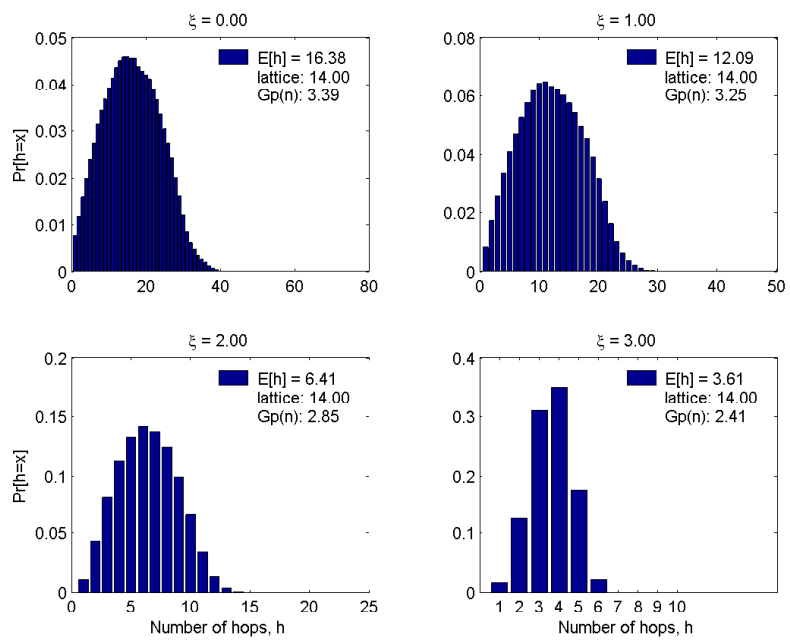

Fig. 7. Hop count in an ad-hoc network for different values of $\xi$. In all subplots $n=1000$, and service area is $20 \times 20$. On each subplot the mean value of the hop-count is indicated together with the mean hop-count in a lattice of size $21 \times 21$ and in a random graph with 1000 nodes and the same link probability as in the ad-hoc network.

\section{CONCLUSIONS}

In this paper we have suggested to use the log-normal shadowing radio model, which is more realistic than the commonly used path-loss model to find the link probability between nodes in wireless ad-hoc networks. With this radio model, the link probability can be expressed in closed mathematical form, as shown in (3).

Through a combination of mathematical modeling and simulations we have shown that in wireless ad-hoc networks, with uniform distribution of nodes over the service area, the degree distribution is binomial if the concentration of nodes around the borders of the service area is low. Compliance with this condition can be checked based on the value of the mean degree. The mean degree, $E[d]$ is calculated with (5). Our simulations show that for square-sized service areas degree distribution can be considered to be binomial when $E[d] \lesssim 18$.

The other fundamental property of ad-hoc networks studied here is the hopcount. We have shown that the hopcount in wireless ad-hoc networks can shift between the hopcount values expected for lattice networks and the hopcount values expected in random graphs. Towards which of these two extremes the actual hopcount leans depends on factor $\xi$ of the radio model. At low values of $\xi$, the men hopcount is close to the mean hopcount in a lattice with the same length and the same width as the service area. As $\xi$ increases, more fluctuations in power loss over radio links can be expected. These fluctuations may cause nodes at long distances to become visible to each other. Consequently, when $\xi$ increases, the mean hopcount tends more towards the mean hopcount in random graphs.

\section{APPENDIX}

Consider $n$ nodes uniformly distributed over a certain twodimensional area $\Omega$. We split area $\Omega$ into $m$ small area's of size $\Delta \Omega$. Assuming that $\Delta \Omega$ is small enough to include only one node, the total number of configurations that can be formed 
with $n$ nodes over the whole area is $\left(\begin{array}{l}n \\ m\end{array}\right)$. We denote these configurations by $L_{1} \ldots L_{\left(\begin{array}{c}m \\ n\end{array}\right)}$. The average number of links over all possible configurations is by definition the number of links in each configuration multiplied by the probability of occurrence of that configuration:

$$
\begin{aligned}
E[L] & =\operatorname{Pr}\left\{L_{1}\right\} L_{1}+\operatorname{Pr}\left\{L_{2}\right\} L_{2}+\ldots .+\operatorname{Pr}\left\{L_{\left.\left(\begin{array}{c}
m \\
n
\end{array}\right)\right\} L_{\left(\begin{array}{c}
m \\
n
\end{array}\right)}}\right. \\
& =\sum_{s=1}^{\left(\begin{array}{c}
n \\
m
\end{array}\right)} \operatorname{Pr}\left\{L_{s}\right\}\left[\sum_{i=1}^{n} \sum_{j=i+1}^{n} p\left(\left|\Delta \Omega_{s, i}-\Delta \Omega_{s, j}\right|\right)\right] .
\end{aligned}
$$

Here $\Delta \Omega_{s, x}$ indicates the position of the placeholder containing node $x$ in configuration $s$, and $\left|\Delta \Omega_{s, i}-\Delta \Omega_{s, j}\right|$ is the distance between two nodes $i$ and $j$ in configuration $s$. This formula can be simplified and rearranged by taking the following into account:

- $n$ nodes can be placed in $m$ possible positions in $\left(\begin{array}{l}m \\ n\end{array}\right)$ distinct ways. If nodes are uniformly distributed over area $\Omega$, all configurations are equally probable with probability $\left(\begin{array}{c}m \\ n\end{array}\right)^{-1}$.

- In the summation over all possible configuration possibilities, each node could be positioned in any of the $m$ possible positions. Therefore, the sum of the link functions $p($.$) over all possible links between n$ nodes over all possible configuration, can be written as summation of link functions $p($.$) over all combination of$ placeholders themselves. Further, we notice that the link function between any two placeholders $i$ and $j$ occurs exactly $\left(\begin{array}{c}m-2 \\ n-2\end{array}\right)$ times (if positions $i$ and $j$ are occupied, there are $n-2$ nodes to be positioned in $m-2$ places, and this can be done in $\left(\begin{array}{c}m-2 \\ n-2\end{array}\right)$ ways).

Considering these points, the formula for $E[L]$ can be rewritten as:

$$
\begin{aligned}
E[L] & =\frac{\left(\begin{array}{c}
m-2 \\
n-2
\end{array}\right) \sum_{i=1}^{m} \sum_{j=i+1}^{m} p\left(\left|\Delta \Omega_{i}-\Delta \Omega_{j}\right|\right)}{\left(\begin{array}{l}
m \\
n
\end{array}\right)} \\
& =\frac{n(n-1)}{m(m-1)} \sum_{i=1}^{m} \sum_{j=i+1}^{m} p\left(\left|\Delta \Omega_{i}-\Delta \Omega_{j}\right|\right) \\
& =\frac{n(n-1)}{m(m-1)} \sum_{i=1}^{m} \sum_{j=i+1}^{m} p\left(r_{i j}\right),
\end{aligned}
$$

where $r_{i j}$ is the distance between two positions $i$ and $j$. We mention here that the above double summation can be simplified in several ways to make numerical computations faster. One method is to rearrange and regroup terms so that summations will be over the number of nodes, rather than number of placeholders. An integral expression is also possible [16].

\section{REFERENCES}

[1] "IETF mobile ad-hoc networks (MANET) working group web site." http://www.ietf.org/html.charters/manet-charter.html.

[2] E. Royer and C.-K. Toh, "A review of current routing protocols for adhoc mobile wireless networks," IEEE Personal Communications, vol. 6, April 1999.

[3] J. Diaz, J. Petit, and M. Serna, "Random geometric problems on $[0,1]^{2}$," Randomization and Approximation Techniques in Computer Science, vol. 1518 of Lecture Notes in Computer Science, pp. 294-306, 1998. Springer-Verlag Berlin.

[4] G. Nemeth and G. Vattay, "Giant clusters in random ad hoc networks," cond-mat/0211325, Nov. 2002.

[5] M. E. J. Newman, S. H. Strogatz, and D. J. Watts, "Random graphs with arbitrary degree distributions and their applications," cond-mat/0007235, May 2001.

[6] M. D. Penrose, "On k-connectivity for a geometric random graph," Random Structures and Algorithms, vol. 15, pp. 145-164, 1999.

[7] C. Bettstetter, "On the minimum node degree and connectivity of a wireless multihop network," Proc. 3rd ACM International Symposium on Mobile Ad Hoc Networking and Computing (MobiHoc), Lausanne, Switzerland, pp. 80-91, June 9-11 2002.

[8] R. Hekmat and P. Van Mieghem, "Interference in wireless multi-hop ad-hoc networks," Proc. Med-hoc-net 2002 Conference, Sardegna, Italy, Sept. 4-6.

[9] M. Ilyas, ed., The Handbook of Ad hoc Wireless Networks. CRC Press, December 2002.

[10] T. Rappaport, Wireless Communications, Principles and Practice. Upper Saddle River Prentice-Hall PTR, 2002.

[11] B. Bollobas, Modern Graph Theory. Springer-Verlag New York, 1998.

[12] R. Albert and A. L. Barabasi, "Statistical mechanics of complex networks," cond-mat/0106096, June 2001.

[13] M. Newman, D. Watts, and S. Strogatz, "Random graph models of social networks," Proc. Natl. Acad. Sci. USA 99, pp. 2566-2572, 2002.

[14] R. Prasad, Universal Wireless Personal Communications. Artech House Publishers, 1998.

[15] L. Sachs, Applied Statistics, a handbook of techniques. Springer-Verlag New York, second edition, 1982.

[16] P. Van Mieghem, "Paths in the simple random graph and the waxman graph," Probability in the Engineering and Informational Sciences (PEIS), vol. 15, pp. 535-555, 2001. 